\title{
Chronic lumbar extradural haematoma simulating disc syndrome
}

\author{
K. V. DEVADIGA AND H. H. GASS \\ From the Department of Neurosurgery, Sinai Hospital and Wayne State University \\ Medical School, Detroit, Michigan U.S.A.
}

SUMMARY Two patients presenting with a clinical picture of nerve root compression suggestive of the herniated disc syndrome are reported. In both the diagnosis proved to be chronic encapsulated extradural haematoma. Recognition of this entity at the time of surgical exposure should render the surgical dissection easier. The postoperative results in both were excellent. One of these patients was on chronic anticoagulant therapy and the unusual location and good prognosis of extradural haematoma in the lumbar spinal canal in such patients is emphasized. In the other, the haematoma developed after a minor back injury, which introduces medico-legal implications.

The occurrence of spontaneous haemorrhage in the spinal extradural space, though infrequent, is now a classifiable pathological entity. Most of the reported 54 cases (Jackson, 1869; Reid and Kennedy, 1925; Shenkin, Horn, and Grant, 1945; Sadka, 1953; Ainslie, 1958; Lowery, 1959; Cooper, 1967; Markham, Lynge, and Stahlman, 1967; Harris, 1969; Rathe, 1969; Boyd and Pear, 1972) have been above the cauda equina level producing cord compression acutely, with profound neurological deficit. When the haemorrhage is in the lumbar area a cauda equina syndrome with radicular symptoms develops. Eight such cases have been reported and four of those (Hopkins, 1899; Chavany, Taptas, and Pecker, 1949; Schultz, Johnson, Brown, and Mosberg, 1953; Pomme, Montrieul, and Girard, 1959) had an acute paraplegic onset. The fact that such $\mathrm{a}^{\text {. }}$ haemorrhage may lead to encapsulated haematoma formation in the lumbar spinal canal and result in a chronic nerve root syndrome simulating herniated disc has been disclosed in the other four reported cases (Svien, Adson, and Dodge, 1950; Harris, 1969; Boyd and Pear, 1972) and reemphasized in the two additional cases described here. One of our cases occurred after a minor back injury and the other in a patient on anticoagulant therapy. The preoperative diagnosis in each was nerve root compression by a herniated disc.

\section{CASE 1}

Patient J.C., a 41 year old male was admitted to Sinai Hospital on 2 June 1970 with low backache and right-sided sciatica. He was in good health until about six months before admission when he strained his back while lifting and developed backache as well as right sciatic pain. He recovered completely and returned to work in four days. About three weeks before admission while pulling a crate he bumped into an object with his back and the severe backache recurred. A week later the pain began to radiate to his buttock, back of right thigh and leg down to his ankle. The pain was almost continuous and made worse by acts of straining. There was no paraesthesia or similar pain on the opposite side.

On examination his gait and stance were normal. Hyperextension and forward flexion of the spine aggravated his sciatic pain. There was restriction of straight leg raising on the side of pain. The right ankle jerk was diminished and there was depression of sensation corresponding to the S1 dermatome. There was also some weakness of right gluteal muscles as compared with the left. The only positive finding in the general physical examination was the presence of urethral discharge.

The plain radiographs of the spine were within normal limits. The lumbar myelogram showed a large extradural defect opposite L5-S1 vertebrae on the right side (Fig. 1).

The patient was treated for acute gonococcal urethritis and after recovery was operated upon on 10 June 1970 . The 5th lumbar and 1st sacral verte- 


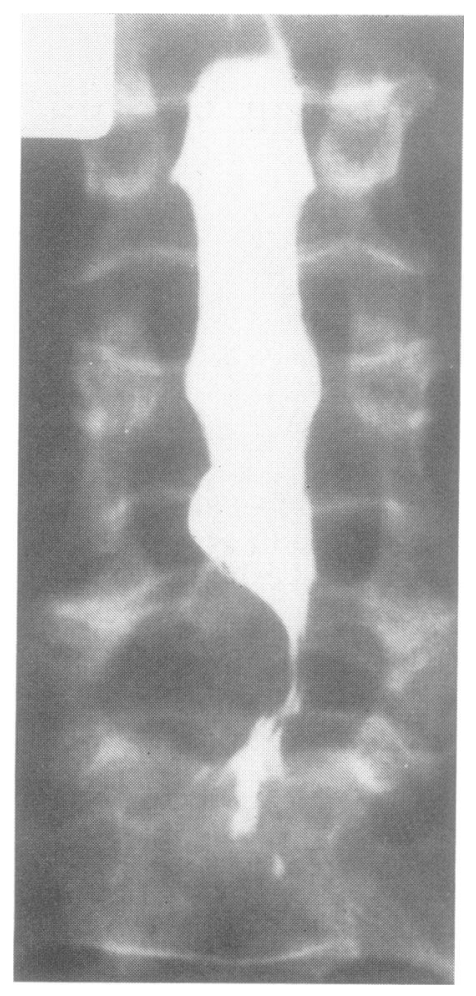

FIG. 1. Case 1. Myelographic defect at L5-SI, right.

brae were exposed on the right side. The ligamentum flavum seemed quite thin. On removal of the ligamentum the exposed dura mater was seen to be discoloured with old blood and it was difficult to identify the S1 nerve root. There was a prominent outpouching of the dura mater opposite the 5th lumbar and 1st sacral vertebrae. There was no evidence of a protruded disc and it was felt that the lesion in this case was something other than a disc. A bilateral laminectomy of L5 vertebra was performed and part of the first sacral vertebra was also nibbled off. It was possible to develop a plane of cleavage between the outpouching of the dura and the normal dura mater, both of which were discoloured by old blood. This exposed a haematoma cavity about the size of a walnut containing old clotted blood. The haematoma capsule was identified and gently teased away from the dura mater. At its attachment to the normal dura mater there was rapid and profuse bleeding from an epidural varix. The bulk of this was lying in the anterior part of the spinal canal. After isolating the first sacral nerve root the surrounding fibrous tissue and haematoma capsule were removed in their entirety.
The histopathological study of the specimen showed broad bands of fibrous tissue containing abundant collagen. In some areas there was a large quantity of haemosiderin and the total appearance was consistent with the diagnosis of an organizing haematoma.

The postoperative period was uneventful. He was last seen on 12 January 1971 , at which time he had mild backache but was relieved of his radicular symptoms.

\section{CASE 2}

Patient K.T., a 65 year old female, was admitted to Sinai Hospital of Detroit on 23 January 1972 because of dragging of her right foot. She had first had back trouble about eight years earlier. This was associated with left-sided sciatica and she was off work for nearly three months. Later in that year she was on traction for three weeks for recurrence of backache. She had been free of back pain until the last week of July 1971 when during the middle of one night she suddenly woke up with severe pain in her right leg. This recurred two or three times during the night and she was admitted two days later on 28 July 1971 to another hospital in Detroit witto persistent pain. It was relieved by bed rest and pelvię traction and she was discharged with minimal pairo along the course of the sciatic nerve. About four weeks before admission she started to drag her right foot. This neurological complaint was not associated with backache or sciatica. She was on long-term anticoagulant therapy for myocardial infarction and angina pectoris.

Neurological examination revealed a steppage gait on the right side. She had a complete range of movement involving the spine. Straight leg raising was not limited. There was a sensory depression corresponding to the L5 dermatome with weakness of dorsiflexion of the right foot. Routine laboratory tests were within normal limits. Plain radiographs of the spine showed narrowing of L4-5 and L5-S1 intervertebral spaces. Myelography showed a lateral defect opposite the last lumbar space on the right side (Fig. 2).

The anticoagulant therapy was discontinued and she was operated upon on 26 January 1972. A right hemilaminectomy of L5 vertebra was done. The S1 nerve root appeared normal. There was no significant disc protrusion at the lumbosacral disc space. However, at the upper border of the L5 lamina there was an ill-defined mass, lateral to the dura mater but close to it. The L5 nerve root was barely visualized as it was hidden under the discoloured mass. The latter was opened at its summit revealing the presence of a cavity containing dark altered blood 
which was completely evacuated. The haematoma capsule was then removed piecemeal from its attachment to the dura mater medially and the nerve root laterally. Inspection of the L4-5 intervertebral disc space did not reveal the presence of a disc protrusion. There was no evidence of abnormal veins in the epidural region.

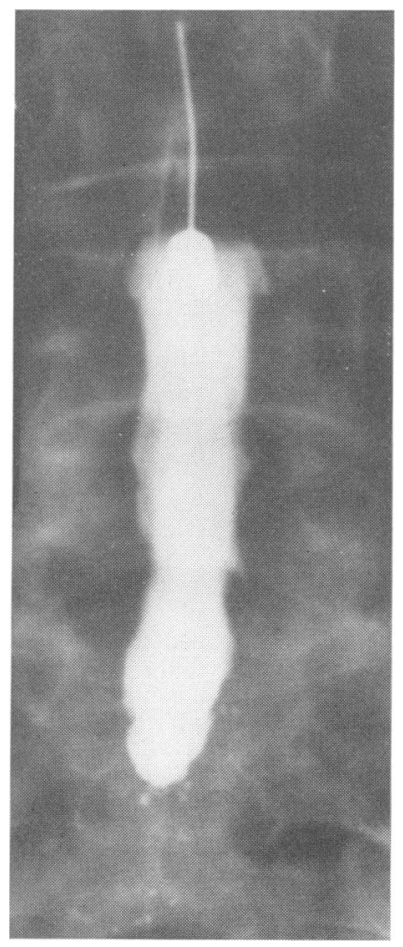

FIG. 2. Case 2. Myelographic defect at L5-S1, right.

Histopathologically the specimen removed consisted of organizing blood clot and fragments of fibro-adipose tissue with granulation tissue reaction.

Her postoperative period was uneventful. The foot drop was corrected with a brace and she was discharged home. When last seen three months postoperatively her foot drop was no longer present and she was free of pain.

\section{DISCUSSION}

The clinical picture of spinal extradural haemorrhage above the lumbar level is characterized by the sudden onset of pain, motor weakness, and urinary retention. The neurological deficit is rapidly progressive and in most of the reported cases paraparesis was noted within three hours after the onset of the initial pain. In the lumbar region the symptomatology is somewhat variable, although acute paraplegia still remains the commonest presenting symptom. In the case reported by Svien et al. (1950), there was a delayed onset of symptoms. In the case of Harris (1969) and the two cases reported by Boyd and Pear (1972), the onset had been an insidious one. In our first case the patient developed sciatica while lifting a weight and recovered completely. The symptoms recurred five months later, three weeks before admission, after another episode of minor trauma. The second case was characterized by an acute onset of leg pain, completely subsiding in a few weeks. Thus, in both cases the onset was mainly with radicular symptomatology.

The aetiology of 'spontaneous' extradural haemorrhage remains speculative. Nevertheless, certain facts are known: (1) the bleeding is mainly venous in origin; (2) the vertebral veins are without valves; (3) the pressure in these veins is lower than in the caval system; and (4) reversal of blood flow occurs in circumstances associated with increased intra-abdominal pressure. Hypothetically, rupture of a weakened epidural vein could occur with a sudden and massive reversal of blood flow from the larger caval system into the smaller vertebral veins. The history of some straining effort in most of the reported cases lends some support to the above hypothesis. However, the relative rarity of epidural haemorrhage in countless numbers of individuals subjected to the common phenomenon of raised intra-abdominal pressure would militate against this as the sole cause of spontaneous epidural haemorrhage. In the case reported by Cooper (1967) the patient was a gymnast and the precipitating factor was not one of the strenuous practice sessions, but a mere strain during micturition. The occurrence of epidural haemorrhage even at sleep as in case 2 and others (Lougheed and Hoffman, 1960; Markham et al., 1967) further invalidates the argument in favour of raised intra-abdominal pressure as the sole factor in the causation of epidural haemorrhage of spontaneous origin. Spinal extradural vascular malformation may be an important additional factor (Nichols and Manganiello, 1956; Maxwell and Puletti, 1957; Cube, 1962; 
Dawson, 1963), as in our first case where there was definite evidence of an epidural varix lying in the anterior part of the spinal canal. The literature dealing with the spinal epidural haemangiomas (Guthkelch, 1948; Brion, Netsky, and Zimmerman, 1952; Newman, 1958), however, does not indicate an association between the two. It is conceivable that the failure to visualize the malformations in spontaneous extradural haemorrhage could be related to the small size of these anomalies or eventual thrombosis after the initial episode of bleeding.

In our second case the epidural haemorrhage was apparently related to anticoagulant therapy, though this particular complication is extremely rare (Cloward and Yuhl, 1955; Alderman, 1956; Winer, Horenstein, and Starr, 1959). Devanney and Osher (1952), at the clinicopathological meeting of Cincinnati General Hospital, presented a patient 62 years of age who was on anticoagulant therapy for thrombophlebitis of the leg, who developed severe backache, bilateral posterior radicular pain, which progressing gradually resulted in paraparesis and urinary retention. The extradural haemorrhage in this case extended from L2 to L5 vertebrae. The patient made a good recovery. Alderman (1956) reported a 57 year old male patient who developed severe pain when he rose from a chair. He was on anticoagulant therapy as a prophylaxis against recurrent myocardial infarction. The pain improved over a period of days only to recur later with a radiating character and ending up with paraparesis. At operation the extradural haemorrhage extended from $\mathrm{Ll}$ to $\mathrm{L3}$ vertebrae.

Even though both of the cases cited above primarily involved the cauda equina region, a severe neurological deficit developed. In our second patient the first episode of pain eight years before this hospitalization was possibly caused by a disc because at that time she was not on anticoagulant therapy. The unique features in this case were the extremely localized nature of the extradural haemorrhage, the mild symptoms, and chronicity, which suggested the diagnosis of recurrent disc herniation. This is in marked contrast with most of the cases hitherto reported in the medical literature.

Because of the greater capacity of the lumbar spinal canal and the ability of the spinal nerves to withstand pressure better than the cord and the relative rarity of impairment of arterial supply to the nerve roots a uniformly good prognosis can be offered in cases of extradural haemorrhage involving the cauda equina region.

A clinical diagnosis of extradural haemorrhage may be entertained, as in the case of Alderman (1956), when the symptoms are fairly typical in a patient who is on anticoagulant therapy. The prognosis in such cases greatly depends upon the rapidity with which the pressure on the cord is relieved. In patients with chronic monoradicular symptoms the diagnosis of extradural haemorrhage, even while the patient is on anticoagulant therapy, has hitherto not been seriously considered. The myelogram in these patients in the presence of a predominantly posterior defect might suggest a different aetiology such as hypertrophic articular facets, thickened ligamentum flavum, or even a needle defect.

\section{REFERENCES}

Ainslie, J. P. (1958). Paraplegia due to spontaneous extro dural or subdural haemorrhage. British Journal of Surgery 45, 565-567.

Alderman, D. B. (1956). Extradural spinal-cord hematomar. report of a case due to dicumarol and review of the literature. New England Journal of Medicine, 255, 838-842. w

Boyd, H. R., and Pear, B. L. (1972). Chronic spontaneous spinal epidural hematoma. Report of two cases. Journal of Neurosurgery, 36, 239-242.

Brion, S., Netsky, M. G., and Zimmerman, H. M. (1952). Vascular malformations of the spinal cord. Archives of Neurology and Psychiatry, 68, 339-361.

Chavany, J. A., Taptas, J. N., and Pecker, J. (1949). A propos d'une variété exceptionnelle d'hématorachis; l'hématome épidural spontané. Presse Médicale, 57, 869-871.

Cloward, R. B., and Yuhl, E. T. (1955). Spontaneous intraspinal hemorrhage and paraplegia complicating dicumarol therapy. Neurology (Minneapolis), 5, 600-602.

Cooper, D. W. (1967). Spontaneous spinal epidural hematoma. Case report. Journal of Neurosurgery, 26, 343-345.

Cube, H. M. (1962). Spinal extradural hemorrhage. Journal of Neurosurgery, 19, 171-172.

Dawson, B. H. (1963). Paraplegia due to spinal epidural haematoma. Journal of Neurology, Neurosurgery, and Psychiatry, 26, 171-173.

Devanney, J. W., and Osher, D. cited by Aring, C. D. (1952). Neurological clinical pathological conference of the Cincinnati General Hospital, Case No. J.H.-8421. Diseases I of the Nervous System, 13, 53-60.

Guthkelch, A. N. (1948). Haemangiomas involving the spinal epidural space. Journal of Neurology, Neurosurgery, $\mathrm{N}$ and Psychiatry, 11, 199-210.

Harris, M. E. (1969). Spontaneous epidural spinal hemor- N rhage. American Journal of Roentgenology, Radium Therapy and Nuclear Medicine, 105, 383-385.

Hopkins, S. D. (1899). Extradural spinal meningeal haemorrhage. With report of a case. New York Medical Journal, $Q$ 70, 296-297. 
Jackson, R. (1869). Case of spinal apoplexy. Lancet, 2, 5-6. Lougheed, W. M., and Hoffman, H. J. (1960). Spontaneous spinal extradural hematoma. Neurology (Minneapolis), 10, 1059-1063.

Lowrey, J. J. (1959). Spinal epidural hematomas. Experiences with three patients. Journal of Neurosurgery, 16, 508-513.

Markham, J. W., Lynge, H. N., and Stalham, G. E. B. (1967). The syndrome of spontaneous spinal epidural hematoma. Report of three cases. Journal of Neurosurgery, 26, 334-342.

Maxwell, G. M., and Puletti, F. (1957). Chronic spinal epidural hematoma in a child. Neurology (Minneapolis), 7, 596-600.

Newman, M. J. D. (1958). Spinal angioma with symptoms in pregnancy. Journal of Neurology, Neurosurgery, and Psychiatry, 21, 38-41.

Nichols, P., Jr., and Manganiello, L. O. J. (1956). Extradural hematoma of the spinal canal. Report of a case. Journal of Neurosurgery, 13, 638-640.

Pommé, B., Montrieul, B., and Girard, J. (1959). Hématome extra-dural lombaire spontané. Revue Neurologique, 101, 69-71.
Rathe, S. (1969). Spontaneous spinal epidural haemorrhage. Journal of the Indian Medical Association, 52, 222-223.

Reid, J., and Kennedy, J. (1925). Extradural spinal meningeal haemorrhage without gross injury to spinal column. British Medical Journal, 2, 946.

Sadka, M. (1953). Epidural spinal haemorrhage with report on 2 cases. Medical Journal of Australia, 2, 669-672.

Schultz, E. C., Johnson, A. C., Brown, C. A., and Mosberg, W. H., Jr. (1953). Paraplegia caused by spontaneous spinal epidural hemorrhage. Journal of Neurosurgery, 10, 608-616.

Shenkin, H. A., Horn, R. C., Jr., and Grant, F. C. (1945). Lesions of spinal epidural space producing cord compression. Archives of Surgery, 51, 125-146.

Svien, H. J., Adson, A. W., and Dodge, H. W., Jr. (1950). Lumbar extradural hematoma. Report of case simulating protruded disk syndrome. Journal of Neurosurgery, 7, 587588.

Winer, B. M., Horenstein, S., Starr, A. M. (1959). Spinal epidural hematoma during anticoagulant therapy. Circulation, 19, 735-740. 